\title{
An alternative floating element design for skin-friction measurement of turbulent wall flows
}

\author{
M. Aguiar Ferreira ${ }^{1}$ - E. Rodriguez-Lopez ${ }^{1}$ B. Ganapathisubramani ${ }^{1}$
}

Received: 6 June 2018 / Revised: 17 August 2018 / Accepted: 20 August 2018 / Published online: 24 September 2018

(C) The Author(s) 2018

\begin{abstract}
Indirect methods to estimate surface shear stress are commonly used to characterise rough-wall boundary-layer flows. The uncertainty is typically large and often insufficient to carry out quantitative analysis, especially for surface roughness where established scaling and similarity laws may not hold. It is, thus, preferable to rely instead on independent measurement techniques to accurately measure skin friction. The floating element was one of the first to be introduced, and still is the most popular for its features. Although its fundamental principle has remained unchanged, different arrangements have been suggested to overcome its inherent limitations. In this paper, we review some of these designs and further present an alternative that is able to correct for extraneous loads into the drag measurement. Its architecture is based on the parallel-shift linkage, and it features custom-built force transducers and a data acquisition system designed to achieve high signal-to-noise ratios. The smooth-wall boundary-layer flow is used as a benchmark to assess the accuracy of this balance. Values of skin-friction coefficient show an agreement with hot-wire anemometry to within $2 \%$ for $R e_{\theta}=4 \times 10^{3}$ up to $10^{4}$. A rough surface of staggered distributed cubes with large relative height, $\delta / h \simeq 10$, is also investigated. Results indicate the flow reaches the fully rough regime, at the measurement location, for the entire range of Reynolds number. Furthermore, the values of skin friction agree with existing estimations from alternative methods.
\end{abstract}

M. Aguiar Ferreira

M.Ferreira@soton.ac.uk

E. Rodriguez-Lopez

E.Rodriguez-Lopez@soton.ac.uk

B. Ganapathisubramani

G.Bharath@soton.ac.uk

1 Aerodynamics and Flight Mechanics Research Group, University of Southampton, Southampton SO17 1BJ, UK 


\section{Graphical abstract}
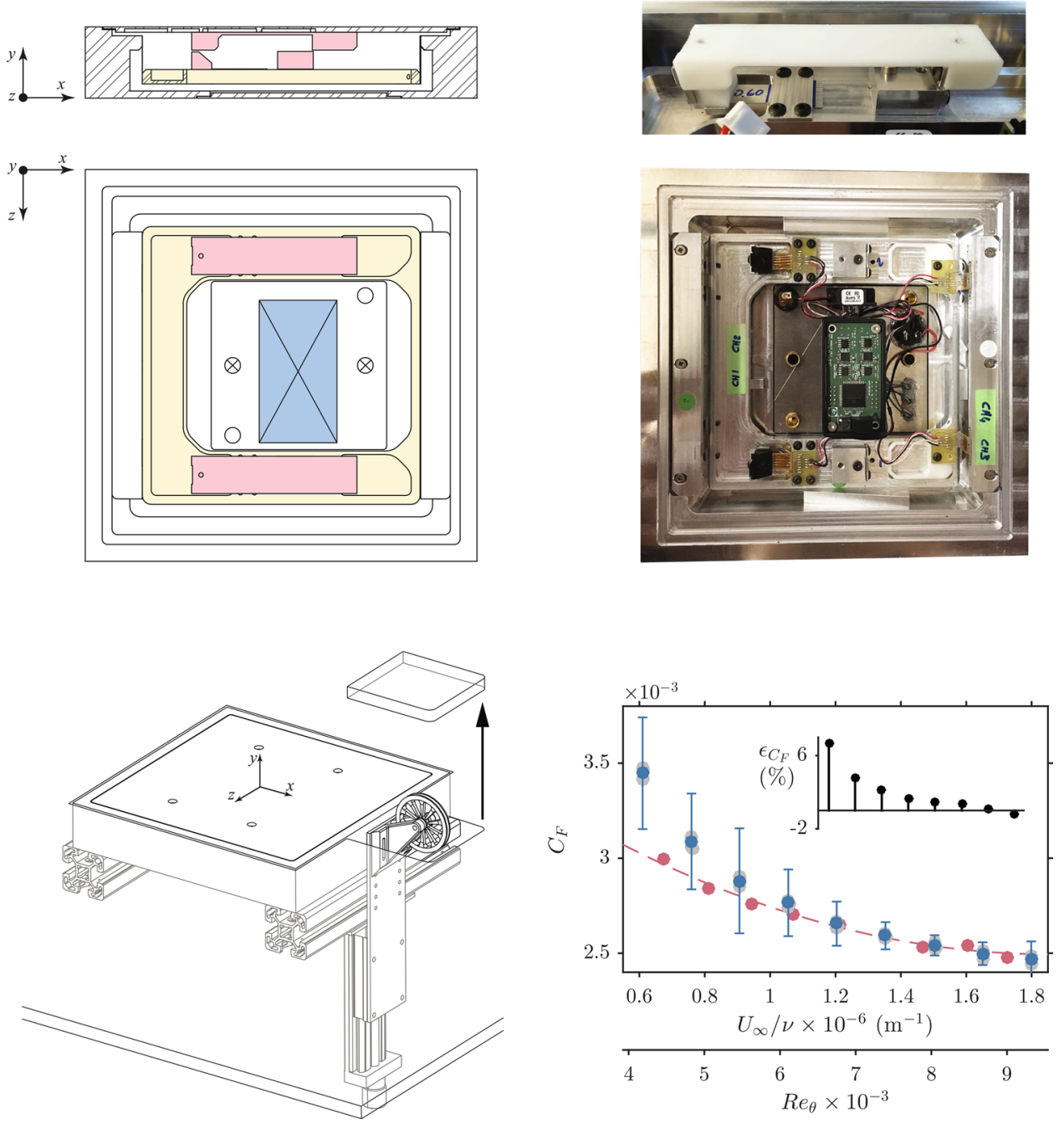

Drawings of the floating-element (FE) balance and skin-friction measurements for a smooth-wall boundary layer. On top: slice along the X-Y plane and top view (left) next to their corresponding pictures (right). Colors highlight distinctsubsystems, namely, the floating frame for drag measurement (yellow), the pitching moment mechanism (red) and the acquisitionsystem (blue). Bottom left: smooth-wall setup and calibration system. The FE is flush mounted with the wind tunnel floor and thepulley is attached to a linear traverse which allows setting its position at different wall-normal locations. During calibration, a lid isremoved to make way for the pulley to move into the test section. A wire is then strung over to suspend the weights. Bottom right: Skin friction over a smooth wall. The inset indicates the relative discrepancy between the FE values (blue) and those inferred fromhot-wire anemometry of the boundary-layer profile (red).

\section{Introduction}

Estimation of the surface shear stress $\tau_{\mathrm{w}}$ is vital for any description of wall bounded flows. It is generally expressed in terms of the friction velocity $U_{\tau}=\left(\tau_{\mathrm{w}} / \rho\right)^{1 / 2}$, which is an important scaling parameter for the structure of turbulent boundary layers. It may alternatively be expressed nondimensionally by the skin-friction coefficient $C_{\mathrm{F}}=\tau_{\mathrm{w}} / q_{0}$, where $q_{0}=\rho U_{0}^{2} / 2$ is the freestream dynamic pressure, $\rho$ is the density of the fluid and $U_{0}$ the freestream velocity. In fact, under certain conditions (Townsend 1976), the mean flow profile and turbulent stresses over smooth surfaces can be solely expressed as a function of $U_{\tau}$ and the length scale $\delta$, the boundary-layer thickness. The friction velocity is then commonly determined via indirect methods, such as the Clauser (1956) chart, by introducing assumptions about the 
flow structure. If the latter are not met, however, the basis of these methods become dubious, and consequently the accuracy of their estimate. This is often the case for boundary layers developing over rough surfaces, where the characteristic roughness height $h$ constitutes an additional length scale which dynamically influences the near wall region (Jiménez 2004). Evidence of such flows, where equilibrium and similarity hypothesis do not hold, is found in the experimental works of Krogstad et al. (1992), Antonia and Krogstad (2001) and Medjnoun et al. (2018), amongst others. Flows past discontinuities in surface roughness are another prime example where indirect methods prove to be inadequate. So much so that seminal works have mostly drawn conclusions based on observations. Quantitative analysis have also been conducted, but in all cases the newly adjusted layer represented a significant fraction of the total boundary-layer thickness (Antonia and Luxton 1971).

Direct measurement techniques, in contrast, benefit from being completely independent of the flow conditions. Some of the most popular include the floating element FE, pressure-based methods and oil-film interferometry, out of which only the first provides the flexibility to carry out experiments with arbitrary surface topologies. Oil film is convenient if the wall shear stress (WSS) is of purely viscous nature (Pailhas et al. 2009; Medjnoun et al. 2018), whereas pressure-based methods apply to rough surfaces that are instead dominated by pressure drag, such as uniform arrays of large two-dimensional ribs or cubes (Antonia and Luxton 1971; Cheng and Castro 2002). The FE principle was introduced by Kempf (1929) nearly a century ago, and since then its use has become widespread. Today, there is a broad variety of FE designs tailored to meet specific requirements. A comprehensive review by Winter (1979) of existing devices reveals many diverse applications: from low-speed to supersonic flows, under distinct pressure gradient, heat and mass transfer conditions. Current designs still share similar features, but rely on more sophisticated signal conditioning systems or large-scale facilities (Baars et al. 2016) to improve the quality of the estimates. In addition, microelectromechanical systems have recently enabled small-scale FE with remarkable frequency response and spatial resolution (Schmidt et al. 1988; Sheplak et al. 2004). Despite their popularity, few studies have actually achieved acceptable levels of uncertainty. Agreement of FE measurements with alternative techniques or established skin-friction laws is generally no better than $5 \%$ for smooth-wall boundary layers. The one of Baars et al. (2016) stands out for achieving a discrepancy level to within $1 \%$ at high Reynolds number, but its large dimensions restrict utility in smaller scale facilities. In this paper, we present a self-contained FE design for studying rough-wall turbulent boundary-layer (TBL) flows, which privileges accuracy and reliability. Its technical aspects are discussed in Sect. 2, followed by a detailed description of the experimental methods in Sect. 3, including calibration and uncertainty analysis. Finally, measurements of skin friction over a smooth and a rough wall are presented in Sect. 4.

\section{The floating element}

Essentially, the FE principle consists in measuring the streamwise net force $F_{\mathrm{w}}$ acting on a finite area $S$ of a boundary-layer plate that is made structurally independent of the rest of the surface. This element is flush mounted, but can move freely in the planar directions facilitated by the existence of an air gap surrounding it. The mean WSS is then obtained via $\tau_{\mathrm{w}}=F_{\mathrm{w}} / S$. FE measurements are subject to a variety of error sources, predominantly of systematic nature, which limit their accuracy and repeatability. There have been few attempts to fully characterise their impact given the practical difficulties to parametrically change the setup of FE and the singular features of each device (O'Donnel 1964; Brown and Joubert 1969; Allen 1977, 1980; Acharya et al. 1985). Consequently, error sources are often quantified by dimensional arguments alone. Here, we review the critical aspects to the design of WSS balances that ultimately determine the quality of their estimates.

\subsection{Design considerations}

Important error contributions arise from the necessary gap surrounding the FE, which makes it sensitive to (i) static pressure differences acting on non-parallel surfaces to the flow. Other sources of uncertainty are associated with (ii) misalignments of the FE relative to the boundary-layer plate and (iii) buoyancy forces resulting from uneven pressure distributions over the flow-exposed surface.

According to Brown and Joubert (1969), contribution (i) is the combination of the direct action of the freestream pressure gradient across the balance, $F_{\alpha}$, and of the cavityinduced flow into and out from the test section, $F_{\mathrm{m}}$. The resultant net force effectively masks out the action of the WSS. They suggest, as a first approximation, that $F_{\alpha} \propto \lambda \rho \alpha S$ and $F_{\mathrm{m}} \propto \rho \gamma U_{\tau} \alpha^{1 / 2} S^{3 / 4}$, where $\alpha=(1 / \rho) \partial p / \partial x$ is the kinematic pressure gradient, $\lambda$ is the lip thickness and $\gamma$ is the nominal gap width, as indicated in Fig. 1. To minimise these loads, we engineered the fixed-to-floating surface joint to a tight tolerance, featuring an S-shaped labyrinth seal $(0.5 \mathrm{~mm}$ thick and $15 \mathrm{~mm}$ long) to artificially tighten the gap. Baars et al. (2016) used instead a grooved-path labyrinth seal that is intrinsically more efficient, though it requires a thicker lip and it may prove difficult to implement. Alternatively, filling the gap with liquid could eliminate unknown pressure forces on the side walls of the FE (the surface tension can be easily quantified). This arrangement is particularly suitable for studies under conditions of non-zero pressure 


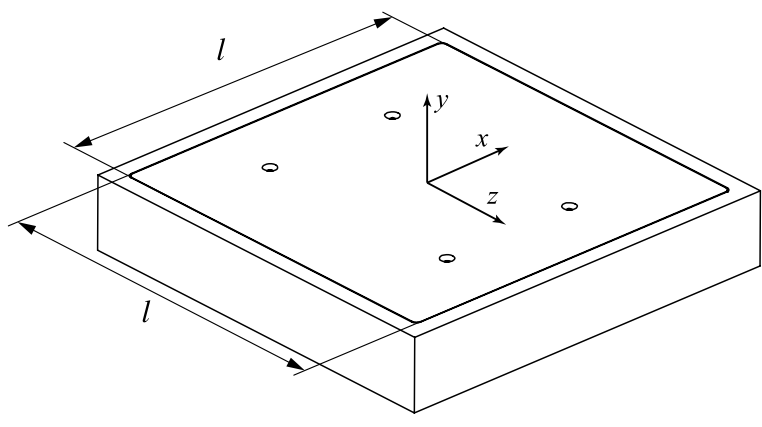

$\gamma$

Detail A
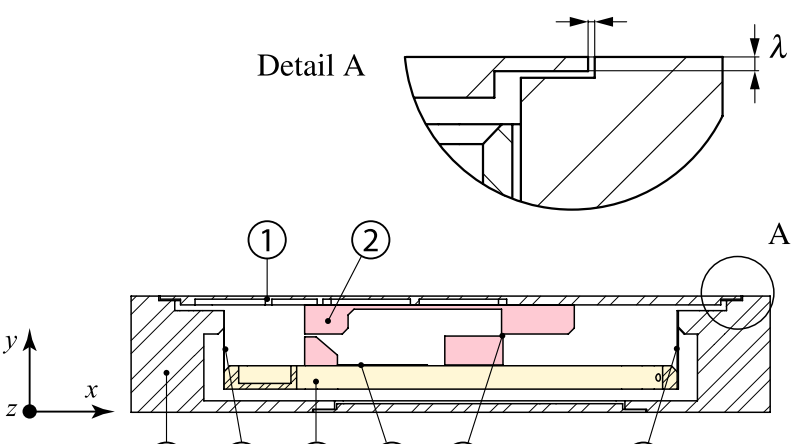

(3)

(4) (5)

(6) (7)

(8)

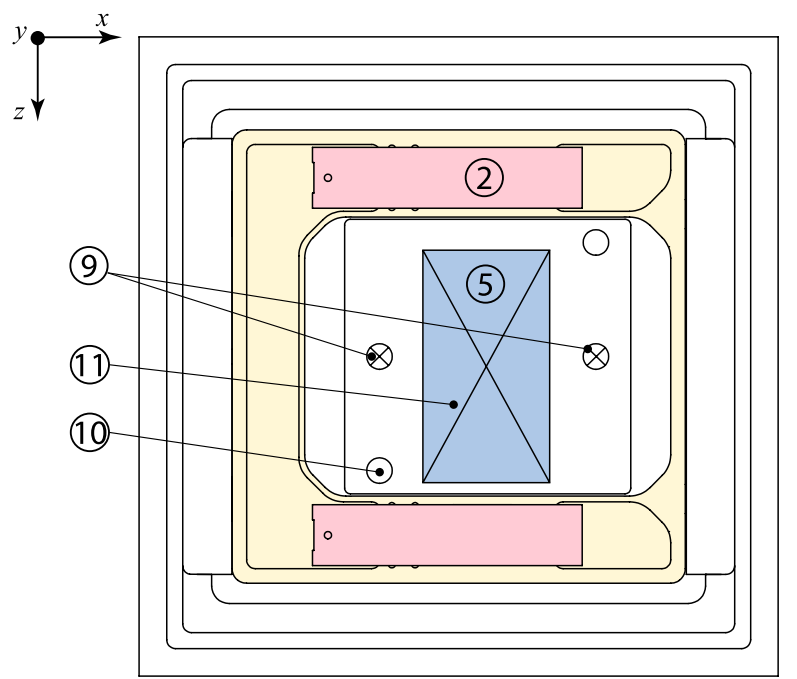

Fig. 1 Drawings of the FE balance. From top to bottom: isometric view with general dimensions, slice along the $X-Y$ plane and top view. (1) Floating element, (2) holders, (3) sealed chamber or casing, (4) flexure, (5) floating frame, (6) pitching moment transducer, (7) pivot point, (8) WSS transducer, (9) outlet to the blower, (10) static pressure taps and (11) acquisition module

gradient (ZPG), such as those carried out by Frei and Thomann (1980) and Hirt et al. (1986), but was discarded for practical reasons. Note, the relationships mentioned above assume the spurious flow is solely driven by the static pressure gradient. They ignore the pressure difference between the flow-exposed surface and the underside of the FE, $\Delta p$, with potentially greater significance. Enclosing the

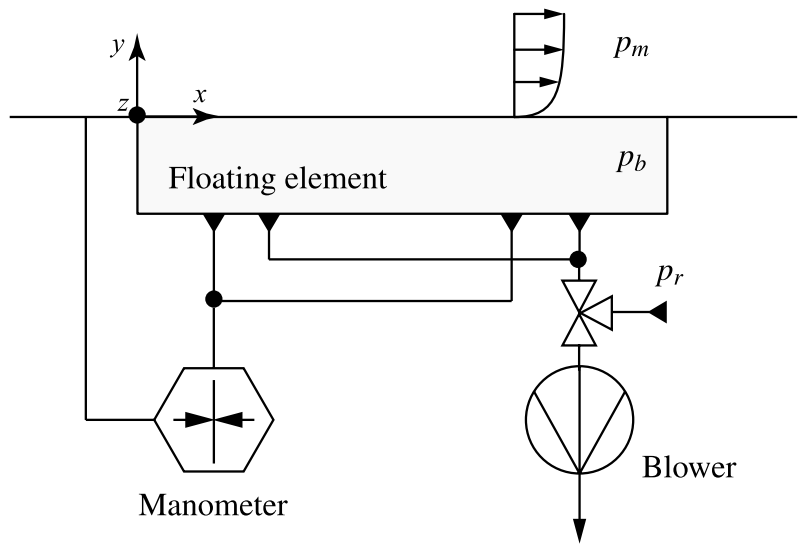

Fig. 2 Active pressure control system. The FE is aligned with the wind-tunnel floor over which a boundary layer grows. $p_{\mathrm{m}}$ is the mean static pressure in the test section, $p_{\mathrm{r}}$ is the room pressure and $p_{\mathrm{b}}$ is the pressure inside the balance.

underside passively contributes towards reducing $\Delta p$, but it is advantageous being able to actively control the pressure differential as well. In the present device, this is achieved by means of a centrifugal blower, as depicted in Fig. 2.

The vertical misalignment of the FE (ii) was initially investigated by O'Donnel (1964) using a single-pivot balance. Years later, Allen $(1977,1980)$ further considered the influence of varying parameters $\lambda$ and $\gamma$, and extended this analysis to parallel-shift designs (the reader is referred to Winter (1979) for a detailed description of both mechanisms). Although his empirical relations may not be directly transferable to other devices, the trends are expected to remain the same. So, they can still be used as guidelines to design balances that are essentially insensitive to misalignments. His results show that, for a fixed protruded or recessed position of the FE relative to the surrounding surface, wider gaps and thinner lips yield smaller lip forces. These act in the direction of a positive drag force and, therefore, should be minimised. Accordingly, the FE comprises a $1 \mathrm{~mm}$-thick flange that extends $10 \mathrm{~mm}$ outward, effectively reducing the lip size, and its edges were rounded off to prevent flow separation. The gap, on the other hand, was primarily designed to negate contribution (i), so it is smaller than would be desirable in terms of sensitivity to misalignments. In any case, the relative position of the FE was optically verified to a resolution of $15 \mu \mathrm{m}$ before each test, by imaging the fixed-to-floating surface joint using a 16-MP camera with high magnification.

Allen (1977) additionally demonstrated how the static pressure distribution over the flow-exposed surface is affected for different protrusion values, causing the centre of pressure to move about. This phenomenon is commonly known as buoyancy (iii) and may have a similar impact to 
that of contributions (i) and (ii) depending on the design of the FE. If simply supported on a single-pivot point (Allen 1977, 1980; Acharya et al. 1985; Krogstad and Efros 2010), the pressure-induced moment is coupled with the moment produced by the surface shear stress-this effect becomes less important if the pivot point is moved farther away from the wall. In contrast, planar-supported elements are naturally insensitive in this regard. One of the first designs of this kind is the parallel-shift linkage, which is equivalent to extending the lever arm to infinity, thereby keeping the floating surface parallel to the flow (Kempf 1929; Dhawan 1951; Coles 1953). The present device is based on this arrangement, as shown in Fig. 1. Other alternatives have also been successfully employed, like placing the FE in a pool of liquid (Frei and Thomann 1980; Hirt et al. 1986) or using air bearings (Ozarapoglu 1973; Baars et al. 2016).

The existence of a fixed-to-floating surface joint entails a perturbation of the boundary-layer flow as it goes over, regardless of all design considerations. Different to contributions (i)-(iii), this error source is of random nature and primarily influences the signal-to-noise ratio (SNR) of the measurement. Here, we evaluate the extent of spurious flow over the FE using the scaling argument introduced by Baars et al. (2016). They argue that for small perturbations the extent of contaminated flow adjacent to the edges of the element is roughly proportional to its perimeter and the gap size or misalignment, whichever is higher. Correspondingly, the ratio of the total surface area to the edge affected area is defined as a proxy for the SNR; it follows from the schematic in Fig. 1 that $\eta_{\mathrm{A}} \simeq l^{2} /(4 \gamma l)$. Large $\eta_{\mathrm{A}}$ values are desirable to improve the quality of the signal. However, practical restrictions in size of wind-tunnel facilities frequently dictate the general dimensions of these devices, and increasing manufacture tolerances as a way to tighten the gap comes with an increase in cost of the project. As a consequence, $\eta_{\mathrm{A}}$ is typically $\mathcal{O}(10)$ and it is highly challenging to achieve larger values. The FE developed by Baars et al. (2016) for the large-scale wind tunnel at the University of Melbourne is notable for having $\eta_{\mathrm{A}}=375$.

\subsection{Mechanical configuration}

The unique design of the present device enables measuring not only the streamwise component of the aerodynamic load but also the induced pitching moment, as a mean to decouple extraneous loads from the measurement of WSS. Its assembly, shown in Fig. 1, consists of a closed aluminium frame with outer dimensions $220 \mathrm{~mm} \times 220 \mathrm{~mm} \times 40 \mathrm{~mm}$ $(W \times L \times H)$, which holds all the mechanical and electronic components inside.

The working principle behind the WSS sensor is similar to that of a parallel-shift linkage. The streamwise load acting on the floating element (1) is directly transmitted to the floating frame below (5), which in turn is supported by a pair of flexures (4) that allow it to move freely along the streamwise direction and a pair of rigid blades (8). These are fixed to the upstream and downstream sides of the casing (3), respectively. The FE is supported by a set of holders (2) which rotate freely about the pivot point (7). The pitching moment generated around it is then counteracted by the pair of blades (6), mounted horizontally on each side of the floating frame. The pivot mechanism consists of a $0.05-\mathrm{mm}$ flexure that is clamped on either sides with a free-standing length of less than $0.5 \mathrm{~mm}$ to prevent it from buckling. Both pairs of blades, (6) and (8), are instrumented with metal gauges for strain measurement, and their sensitivity can be adjusted by varying the effective length. In the current configuration, the vertical transducers are fixed-supported aluminium blades $15 \mathrm{~mm}$ long, $10 \mathrm{~mm}$ wide and $0.45 \mathrm{~mm}$ thick. Those for the pitching moment are instead simply supported, $15 \mathrm{~mm}$ long and $0.6 \mathrm{~mm}$ thick, resulting in greater sensitivity. The sensing element is a $200-\mathrm{mm}$-side square, small enough in relative terms to ensure local measurement of WSS. Its dimensions are such that the variation in friction coefficient $C_{\mathrm{F}}$ is less than $1 \%$ between the leading and trailing edges. The 1/7th law skin-friction relationship was used to obtain estimates of the local $C_{\mathrm{F}}$ for a smooth-wall boundary layer at $R e_{\mathrm{x}} \simeq 2 \times 10^{6}$. This corresponds to a freestream velocity of $10 \mathrm{~ms}^{-1}$ and a development length of approximately $3 \mathrm{~m}$, according to the experimental setup described in Sect. 3. In view of error contributions (i) and (ii) (refer to Sect. 2.1), the fix-to-floating surface joint was carefully designed to a very tight clearance. Detailed in Fig. 1, the lip $\lambda=1 \mathrm{~mm} \pm 0.1$ and the gap $\gamma=0.5 \mathrm{~mm} \pm 0.1$, with a 15-mm-long labyrinth seal which further mitigates cavityinduced flow asymmetries. Finally, the size of the FE and the gap width yield an effective surface area coefficient of $\eta_{\mathrm{A}}=100$. This value is much larger than average and falls in the same order of magnitude of the one from the largescale TBL facility in Melbourne, though its dimensions are significantly smaller in comparison (the area ratio is 75 ).

This balance also features an external centrifugal blower used to modify the pressure inside, $p_{\mathrm{b}}$. There are two nozzles mounted at the bottom, one on each of the two sides of the acquisition module, as depicted in Fig. 1. This is to ensure uniform pressure distribution on the underside of the FE and to minimize airflow inside the balance. $p_{\mathrm{b}}$ is monitored via two pressure taps, included in the same schematic, mounted on opposite corners away from the nozzles. The circuit is detailed in Fig. 2. Both the pressure taps and the ports to the blower inlet are externally connected. The blower is driven by a 24 VDC motor that is controlled through pulse-width modulation and is able to generate up to $1400 \mathrm{~Pa}$ of head pressure. The intensity of the blower can further be adjusted by regulating a three-way valve placed upstream, which lets air from the room to be sucked in. 


\subsubsection{Relevance of the pitching moment}

One of the primary challenges to WSS measurement consists in designing a mechanism that is sensitive to streamwise loads alone. The ability of the system to negate other components is mostly related to geometrical tolerances, and in this particular case to the electrical characteristics of the strain sensors as well. It is virtually impossible to attain perfect orthonormality between the parts that make up the FE. So the pair of force transducers (8), in Fig. 1, may be slightly off the wall-normal direction, and similarly its strain gauges may exhibit a small misalignment between themselves and the blades. The balance is, therefore, not completely insensitive to normal loads, as drag transducers have intrinsically some degree of transverse sensitivity (in the axial direction). Although this effect is ordinarily quite small, it still stands as a source of bias error if $\Delta p$ is not equalised. To circumvent this limitation, we use the pitching moment sensor to control the blower such that the pressure-induced force is cancelled out. This is possible since the pivot point is offset from the centroid of the FE, and provided the buoyancy effects are not significant, in which case the centre of pressure could shift toward the location of the pivot point, hence reducing the moment produced by the vertical load. Furthermore, the latter cannot entirely be eliminated for rough-wall boundary layers as the zero-plane displacement effectively constitutes a lever arm for the WSS. The magnitude of the moment it induces is relatively smaller, yet entails the existence of a non-zero net vertical force to balance it.

\subsubsection{Compliance}

The tight clearance between the floating and fixed surfaces of this device, and the additional complication of an extra degree of freedom demand a thorough examination of the motion of the FE. This is a function of the stiffness of the force transducers, the geometry of the balance and the loads it may likely experience.

In the facility described in Sect. 3, the typical local Reynolds number $R e_{\theta}$ of smooth-wall boundary layers is $\mathcal{O}\left(10^{3}\right)$ (Dogan et al. 2016), for freestream velocities ranging from 10 up to $25 \mathrm{~ms}^{-1}$. Under these conditions, the maximum integrated skin friction over the sensing area is approximately $50 \mathrm{mN}$ from the logarithmic correlation of Österlund et al. (2000), $C_{\mathrm{F}}=2\left((1 / \kappa) \ln \left(R e_{\theta}\right)+4.08\right)^{-2}$. It is less straightforward estimating the WSS over rough walls as it highly depends on its topology. Still, it is safe to assume loads up to two orders of magnitude larger for the rougher cases, $\mathcal{O}(1) \mathrm{N}$. Knowing the dimensions of the force traducers, it is possible to determine their maximum deflection and thereby estimate the corresponding displacement of the element. To illustrate, an integrated shear stress of $1 \mathrm{~N}$ applied $5 \mathrm{~mm}$ away from the wall would displace it along the streamwise direction by $3.5 \mu \mathrm{m}$, and the maximum step height at the leading edge due to inclination of the surface would be $40 \mu \mathrm{m}$. In terms of viscous units $v / U_{\tau}$, where $v$ is the kinematic viscosity of the fluid, the protrusion would be smaller than 3 for the highest freestream velocity, ensuring the FE is hydraulically smooth across its whole range of operation. Note, this analysis neglects any pressure difference between the top and the underside of the FE that is generally kept to a minimum.

\subsection{Data acquisition system (DAQ)}

The ability of the balance to measure WSS depends, amongst other factors, on the integrated wall drag, the sensitivity of the force transducers and the effective resolution of the analog-to-digital converter (ADC). Since practical restrictions in size of the FE limit the magnitude of the force it experiences $\left(F_{\mathrm{w}}=\tau_{\mathrm{w}} S\right)$, an acquisition module able to provide a stable low-noise signal is paramount to ensure the quality of the measurements. In this section, we briefly explain the signal conditioning system especially designed for this application, which involves a four-channel acquisition module and two sets of active strain sensors, one for each of the two load components (drag and pitching moment).

\subsubsection{Force transducers}

The WSS produced by a smooth-wall boundary layer was considered to estimate the required sensitivity of the force transducers and resolution of the ADC. Specifically, one developing in the low-speed wind tunnel described in Sect. 3, at $R e_{\theta}=3830$ and a freestream velocity of $10 \mathrm{~ms}^{-1}$ (Dogan et al. 2016). This is expected to be the most demanding testing condition with $C_{\mathrm{F}}=3.05 \times 10^{-3}$, based on the relationship of Österlund, yielding an integrated skin friction of $F_{\text {w min }} \simeq 7.3 \mathrm{mN}$.

Ideally, the number of noise-free binary quantization levels of the ADC, \#counts, is given by the ratio between the full-scale load, $F_{\mathrm{FS}}$, and the desired resolution. The former was taken to be $\mathcal{O}(1) \mathrm{N}$, which is roughly the maximum bulk drag generated by a rough patch with large relative height, and the minimum resolution is defined as $0.1 \%$ of $F_{\text {wmin }}$; thus, \#counts $=1.37 \times 10^{5}$. Considering the geometry of the FE, the sensitivity to WSS is defined as $\Sigma_{F_{\mathrm{w}}}=2 K \varepsilon_{\mathrm{FS}}=0.1636 \mathrm{mV} / \mathrm{V}$, where $K$ is the Young modulus of the blades and $\varepsilon_{\mathrm{FS}}$ is the mechanical strain under full-scale load. A factor of 2 was introduced since two force transducers are used in parallel. With a 5-V excitation 
voltage, the full-scale output of each drag transducer is $V_{\mathrm{FS}}=(0.1636 / 2) \times 5=0.41 \mathrm{mV}$, yielding a minimum peakto-peak noise-free resolution of $V_{\mathrm{p}-\mathrm{p}}=2.99 \mathrm{nV}$. Assuming normally distributed noise, its root-mean-square (RMS) is estimated from the peak-to-peak value using the coverage factor $k=6.6$, which corresponds to a confidence interval of $99 \% ; V_{\mathrm{RMS}}=V_{\mathrm{p}-\mathrm{p}} / k=0.46 \mathrm{nV}$. The requisites of noise-free and effective resolution were then estimated as

$$
\begin{aligned}
& R_{\text {free }}=\log _{2} \frac{V_{\mathrm{FS}}}{V_{\mathrm{p}-\mathrm{p}}}=\log _{2} \frac{0.41 \mathrm{mV}}{2.99 \mathrm{nV}}=17.07 \\
& R_{\text {eff }}=\log _{2} \frac{V_{\mathrm{FS}}}{V_{\mathrm{RMS}}}=\log _{2} \frac{0.41 \mathrm{mV}}{0.46 \mathrm{nV}}=19.77 .
\end{aligned}
$$

These values indicate that at least a 20-bit $\mathrm{ADC}$ is required to achieve the skin-friction resolution mentioned above. An equivalent analysis was conducted for the pitching moment sensor whose sensitivity is $\Sigma_{F_{\mathrm{w}}}=0.1696 \mathrm{mV} / \mathrm{V}$, similar to that of WSS.

\subsubsection{Acquisition module}

The acquisition module was designed in-house around the integrated circuit AD7195 from analog devices. It is driven by a $5-\mathrm{V}$ power supply and it includes the required signal conditioning, filtering and an ultra-low-noise 24-bit sigma-delta ADC. The bridges on the force transducers were arranged in six-lead assemblies, where the same source is used both as the reference for the digital converter and for exciting the strain sensors. This way, changes to the excitation do not introduce measurement error as the output is ratiometric. An $\mathrm{AC}$ square wave is used to excite the force transducers which, together with the chopping scheme on the ADC, is able to cancel out induced DC errors, effects of parasitic thermocouples, and to average out pink noise that is dominant at low frequencies in these kinds of applications. As a result, the acquisition system is capable of producing discernible output signals with a much lower excitation voltage in comparison to DC-driven sensors, enjoying a much higher SNR. The printed circuit board was dimensioned to fit inside the balance. The wiring from the bridges to the acquisition module were, therefore, kept short and are shielded by the aluminium case, hence reducing electromagnetic interferences. Wind tunnel off, the RMS of the noise is $56 \mathrm{nV} / \mathrm{V}$ and goes up to $190 \mathrm{nV} / \mathrm{V}$ once the drive unit is enabled.

\section{Experimental methods}

The FE is currently employed in the suction-type wind tunnel of the experimental fluid mechanics laboratory at the University of Southampton. This open-return wind tunnel

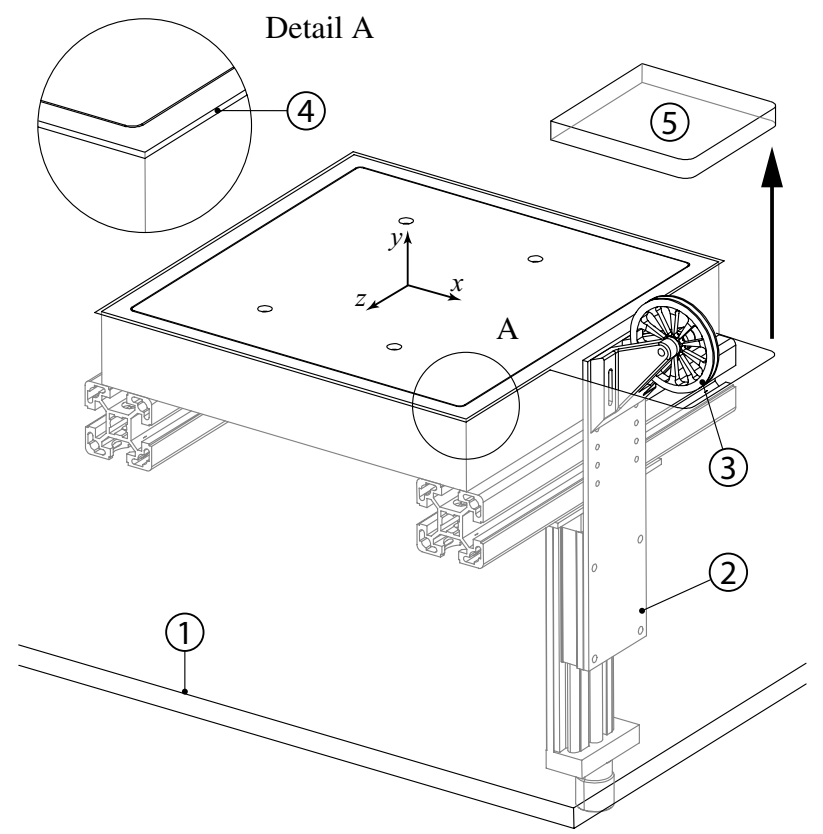

Fig. 3 Smooth-wall setup. The FE is flush mounted with the windtunnel floor (1) and the gap surrounding the balance (4) is taped over to prevent leaks. The pulley (3) is attached to a linear traverse (2) which in turn is bolted onto the main frame. To calibrate, the lid (5) is removed to make way for the pulley to move into the test section. A wire is then strung over to suspend the weights

has a 7:1 contraction followed by a closed working section $4.5 \mathrm{~m}$ long with cross section $0.9 \mathrm{~m}$ wide and $0.6 \mathrm{~m}$ high. The freestream turbulence intensity has been reported to be homogeneous and lower than $0.5 \%$ at the measurement location (Claus et al. 2012; Placidi and Ganapathisubramani 2018). Despite not featuring an adjustable roof, experiments were conducted in nominal ZPG as the acceleration parameter could be neglected; it is on the order $\mathcal{O}\left(10^{-8}\right)$. The walls of the test section are optically transparent to facilitate using imaging techniques, like particle image velocimetry (PIV), and the floor is cut out to fit the FE balance. The setup is illustrated in Fig. 3.

We consider two different kinds or surface topology: a smooth wall, used as the benchmark to assess the viability of this device, and a staggered array of cubes with 0.25 plan solidity fraction. Its relative height $h / \delta \simeq 0.1$, where $h$ is the height of roughness obstacles and $\delta$ is the thickness of the boundary layer. This particular surface roughness has been previously studied by Claus et al. (2012) and Cheng and Castro (2002), who provide direct measurements of WSS using a pivot-type FE and pressure-tapped roughness obstacles, as well as vertical profiles of the normal and shear stresses obtained via cross-wire anemometry. Boundary layers are established directly on the wind-tunnel floor, following the contraction. Before reaching the working section, they experience an intense favourable pressure gradient, which 


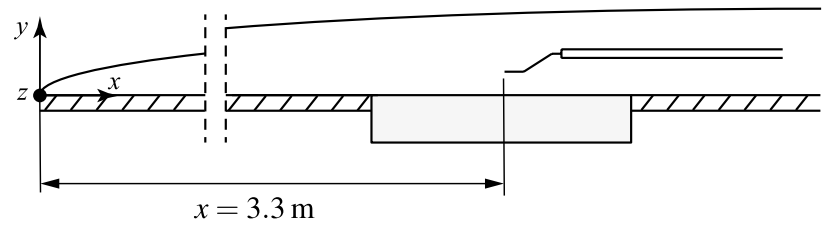

Fig. 4 Smooth-wall and hot-wire arrangement. Measurements were taken at the centreline of the FE, 3.3 m downstream of the test section

in the smooth-wall case undermines the validity of existent empirical relationships for the skin-friction coefficient. Furthermore, tripping conditions have been shown to impact the development of TBL (Rodríguez-López et al. 2016). For these reasons, the local skin friction is instead estimated from a series of hot-wire anemometry of the velocity profile.

\subsection{Hot-wire anemometry}

Hot-wire measurements of the streamwise velocity profile were taken $3.3 \mathrm{~m}$ downstream of the contraction, at the centreline of the FE, as depicted in Fig. 4. A total of 9 freestream velocities equally spaced between 10 and $26 \mathrm{~ms}^{-1}$ were considered.

The boundary-layer probe is an Auspex A55P05 with 10 -mm-long prongs spanned by a 3-mm-long and $5-\mu$ $\mathrm{m}$-diameter tungsten wire. The central active region is approximately $1 \mathrm{~mm}$ long and is shouldered on either side by copper-plated sections, yielding an effective length-todiameter ratio of 200. The wire was operated by a Dantec StreamLine Pro constant temperature anemometer (CTA) system set to an overheat ratio of 1.8. We used the National Instruments USB-6212 BNC ADC to sample the signal at $20 \mathrm{kHz}$. Pre- and post-calibrations were conducted using a Pitot-static tube, mounted at the same streamwise location, that was connected to a Furness FCO510 micromanometer. The temperature was constantly monitored throughout calibration and acquisition stages using a T-type Omega thermocouple. Each velocity profile is populated by 30 points taken for a sampling period of $T=40 \mathrm{~s}$, which in the worst case $\left(U_{0}=10 \mathrm{~ms}^{-1}\right)$ is equivalent to $T \delta / U_{0}=8000$ eddy turnover times.

The mean skin friction was estimated using the method proposed by Rodríguez-López et al. (2015). Accordingly, the mean velocity profile is compared with a canonical description of the boundary layer following Musker (1979), for the inner and logarithmic layers, and using the exponential wake by Chauhan et al. (2009). The method allows adjusting the wall-normal coordinate to overcome any potential uncertainty in determining the initial relative position of the wall probe. The skin friction is then estimated as that which minimises the error between the

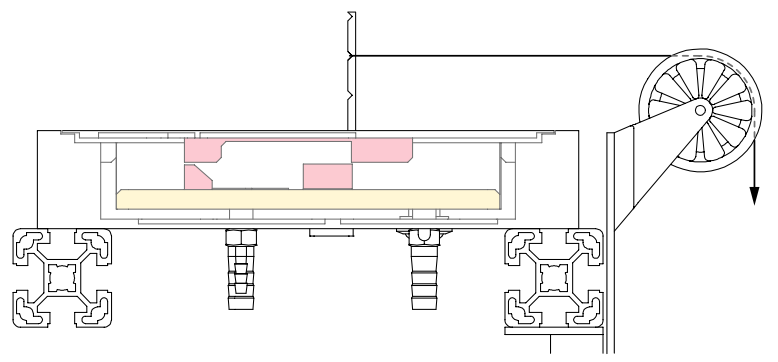

Fig. 5 Typical calibration setup. In this schematic, the see-through casing shows the alignment of the stud with the pivot point. The wire is looped around it, in the middle notch, and goes over the pulley that is mounted on the linear traverse. The nozzles to the centrifugal blower and pressure taps are also visible

experimental and canonical profiles. For further details on this method the reader is referred to the original paper (Rodríguez-López et al. 2015). The accuracy in determining $U_{\tau}$ is better than $1 \%$ for perfectly accurate velocity profiles and better than $0.5 \mathrm{v} / U_{\tau}$ for the initial relative position of the wall probe. The method was originally validated against DNS and experimental databases, under presence of mild pressure gradients, wall probe interference in the inner layer and poorly converged data. Additionally, it has been shown to perform adequately in highly disrupted flows past strong tripping conditions (Rodríguez-López et al. 2016), porous fences (Rodríguez-López et al. 2017) and TBL under freestream turbulence (Esteban et al. 2017).

\subsection{Static calibration}

The balance was statically calibrated in situ using a set of weights and the wire-pulley arrangement pictured in Fig. 5. The pulley is an ME-9450 from Pasco with 50-mm-diameter string and an effective coefficient of friction of $7 \times 10^{-3}$. It was attached to a 50-mm linear traverse which enabled adjusting its vertical position to within $0.05 \mathrm{~mm}$. For calibration purposes, a 40-mm-long stud with machined-in grooves was mounted perpendicularly to the $\mathrm{FE}$ at the spanwise centerline, above the pivot point. A 0.1-mm nylon wire was then looped around it and strung over the pulley. A small mass was suspended to tension the wire, so that its alignment could be adjusted. First, by sliding the traverse system across the spanwise direction and second, by turning the knob to adjust the vertical position of the pulley to a predetermined height.

Prior to any acquisition, the device was turned on and let to warm up for $2 \mathrm{~h}$. The calibration involved recording the response of the strain sensors to $n=24$ load combinations using M1-class weights. The output voltages were sampled for $20 \mathrm{~s}$ to average out noise and the mean values of each pair of transducers were summed up. For the smooth wall, six different weights including the zero reading $(0,1,3,5,10$ and $15 \mathrm{~g})$ were applied at four different heights $(0,9.6,19.2$ and 
$28.8 \mathrm{~mm}$ ). This process was repeated 5 times to achieve statistical significance, so a grand total of 120 calibration points were taken. Note the loading direction of the balance over the course of the calibration changed to account for potential hysteresis. The values of force $F_{\mathrm{w}}(\mathrm{N})$ and pitching moment $M_{\mathrm{y}}$ $(\mathrm{Nm})$ were obtained by multiplying the mass of the weights by the local gravity acceleration $g=9.81084 \mathrm{~ms}^{-2}$, from the land gravity survey data by the British Geological Survey.

We consider the second-order response surface model in two variables:

$y=\beta_{1} x_{1}+\beta_{2} x_{2}+\beta_{11} x_{1}^{2}+\beta_{22} x_{2}^{2}+\beta_{12} x_{1} x_{2}+\varepsilon$.

By letting $x_{3}=x_{1}^{2}, x_{4}=x_{2}^{2}, x_{5}=x_{1} x_{2}, \beta_{3}=\beta_{11}, \beta_{4}=\beta_{22}$ and $\beta_{5}=\beta_{12}$, Eq. 3 is rewritten in matrix form as

$Y=X \beta+\varepsilon$,

where

$Y=\left[\begin{array}{cc}y_{11} & y_{12} \\ {[2 p t] y_{21}} & y_{22} \\ \vdots & \vdots \\ y_{n 1} & y_{n 2}\end{array}\right] ; \quad X=\left[\begin{array}{cccc}x_{11} & x_{12} & \ldots & x_{15} \\ x_{21} & x_{22} & \ldots & x_{25} \\ \vdots & \vdots & \vdots & \\ x_{n 1} & x_{n 2} & \ldots & x_{n 5}\end{array}\right] ; \quad B=\left[\begin{array}{cc}\beta_{11} & \beta_{12} \\ \beta_{21} & \beta_{22} \\ \vdots & \vdots \\ \beta_{51} & \beta_{52}\end{array}\right]$.

$Y_{n \times 2}$ is the calibration design matrix; its rows $i$ refer to different loading conditions and the columns $j$ are for the individual components of the applied load, that is, $F_{\mathrm{w}}$ and $M_{\mathrm{y}}$, respectively. The model matrix, $X_{n \times 5}$, contains information about the response of the force traducers to each load combination (in units of $\mathrm{mV} / \mathrm{V}$ ), as well as information about the model form-the number of columns corresponds to the number of terms in the right-hand side (RHS) of Eq. 3. The regression coefficients $B$ are estimated via weighted least squares (WLS) fit to account for the uncertainty in the predictors. This is particularly important as the size of the dataset is fairly small. From Strutz (2016),

$\hat{B}_{k j}=\left(\bar{X}^{T} W^{j} \bar{X}\right)^{-1} \bar{X}^{T} W^{j} Y$,

where $\hat{B}$ is the least squares estimator of $B, W^{j}$ is the weighting matrix for a particular degree of freedom (DOF), $j$, and the overbar indicates averaged quantities over all calibration runs (five in total). As suggested by Reis et al. (2013), the weighting matrix is computed as the sum of the error contributions due to weights and the wire-pulley $\operatorname{setup} V_{\mathrm{w}}$, such as misalignments and friction forces, and the variance in the readings of the strain sensors $V_{\mathrm{r}}$. Accordingly,

$W^{j}=\left(V_{w}^{j}+D^{j} V_{\mathrm{r}} D^{j^{T}}\right)^{-1}$.

The second term on the RHS of Eq. 6 should be thought of as the projection of the variance in the readings onto the estimated load. $V_{\mathrm{r} 2 n \times 2 n}$ is diagonally symmetric and is computed as the covariance between the readings of the force traducers, whereas $D_{n \times 2 n}^{j}$ is the sensitivity matrix whose diagonal contains the partial derivatives of Eq. 3 with respect to a given DOF. Finally, $V_{w n \times n}^{j}$ is a diagonal matrix and its values are statistically approximated by the sum of squared residuals $\left(\mathrm{SS}_{E}\right)$ of the fit by replacing $W$ for the identity matrix.

The parameters of the linear regression are listed in Eq. 7. They also appear divided by the respective uncertainty $\left|\hat{B} / u_{\mathrm{B}}\right|$ to highlight the relative importance between them. $u_{B}$ is the positive square root of the main diagonal elements of $\left(\bar{X}^{T} W^{j} \bar{X}\right)_{5 \times 5}^{-1}$, which is the covariance matrix of the model coefficients.

$\hat{B}=\left[\begin{array}{cc}F_{\mathrm{w}} & M_{\mathrm{y}} \\ -2.178 & 81.439 \\ 0.002 & -115.143 \\ -0.115 & -91.906 \\ -0.075 & -121.468 \\ 0.124 & 106.961\end{array}\right] ; \quad\left|\frac{\hat{B}}{u_{\mathrm{B}}}\right|=\left[\begin{array}{cc}F_{\mathrm{w}} & M_{\mathrm{y}} \\ 0.02 & 9.09 \\ 0.05 & 0.21 \\ 0.03 & 0.30 \\ 0.05 & 0.26\end{array}\right]$.

The large magnitude of $B_{11}$ in comparison to the remaining terms reveals a strong linear behaviour of the drag sensor, independent of the pitch moment acting on the FE. On the contrary, the latter appears to be equally dominated by the linear and nonlinear terms of both force transducers. The corresponding values of $\left|\hat{B} / u_{\mathrm{B}}\right|$, however, indicate that the relative importance of the second-order terms is negligible as the associated variance is significantly high. This is likely due to lack of orthogonality in the design of the response model (Johnson et al. 2010). The variance inflation factor (VIF) is a statistical indicator that quantifies the degree of multicollinearity in a least squares regression. As a rule of thumb, the value of VIF is ideally kept under ten, but in this particular case is $\mathcal{O}\left(10^{2}\right)$, which is explained by the large cross correlation value between the readings,

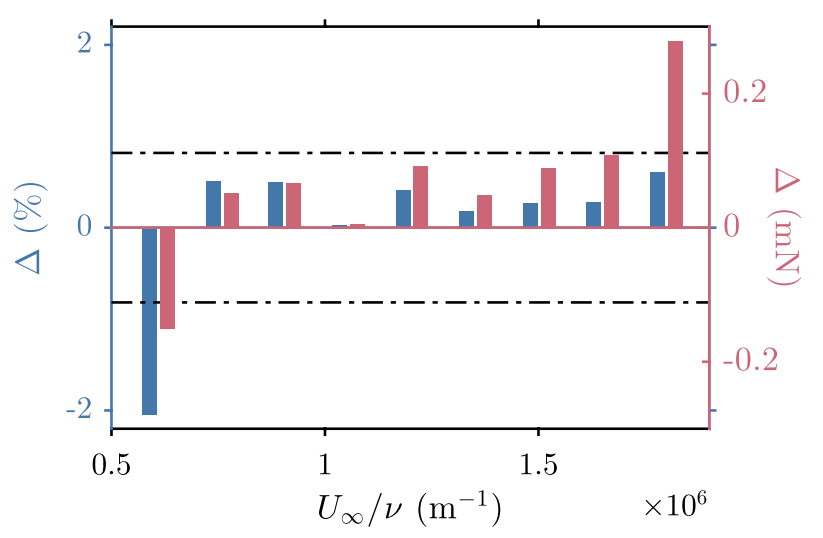

Fig. 6 Long-term stability of the calibration. Relative (left) and absolute (right) discrepancies between measurements of wall drag for the smooth-wall boundary layer case over a range of Reynolds numbers. Drag values were obtained using different calibrations performed 2 weeks ahead of and after the measurements 
$x_{i 1} \otimes x_{i 2}=0.953$. Still, the standard error of the fitting is relatively small: $\hat{\sigma}_{F_{\mathrm{w}}}=0.057 \mathrm{mN}$ and $\hat{\sigma}_{M_{\mathrm{y}}}=0.006 \mathrm{mN} \mathrm{m}$, where $\hat{\sigma}^{2}=\mathrm{ss}_{\mathrm{E}} /(n-5)$.

A series of pre- and post-calibrations were carried out over the course of 1 month to assess the long-term stability of the balance to environmental changes and setup. The difference between measurements of WSS obtained using linear estimators from calibrations performed weeks apart is shown in Fig. 6. Apart form the data point taken at the lowest Reynolds number, discrepancies between calibrations are generally smaller than $0.5 \%$ with a standard deviation of just over $0.8 \%$, or $0.1 \mathrm{mN}$ in absolute terms.

\subsection{Measurement of WSS}

The balance is first turned on and let to warm up for $2 \mathrm{~h}$, unless a pre-off-tunnel calibration was performed before the measurement. In the meantime, the tunnel is ran for an extended period to ensure steady flow conditions. The acquisition sequence is fully automated; it starts with a shakedown period of 3 min during which the wind tunnel is set at the maximum freestream velocity. It is then turned off for half an hour before zeroing the balance. This initial kick guarantees the very first data point is taken under the same conditions as the first data point of subsequent runs. A total of nine drag values were acquired over a range of $R e$ for both the smooth and the rough wall. The evaluation method is illustrated in Fig. 7. For each freestream velocity the pressure inside the balance $p_{\mathrm{b}}$ is progressively varied using the blower such that the pitching moment passes through the zero-crossing. This experiment was conducted in a suction wind tunnel, so the static pressure in the test section is naturally lower than $p_{\mathrm{b}}$. Consequently, given the location of the pivot point, the pitching moment is initially positive but gradually becomes negative with increasing suction of the blower. The values of wall drag are the Y-intercept of the linear regression through each set of data points acquired at fixed $R e$, where the pitching moment is null. This is a necessary step since the extraneous contribution of the vertical load to the measurement of WSS is minimised when the moment it induces is cancelled out, as explained in Sect. 2.2.1. Acquisitions last $30 \mathrm{~s}$ at $150 \mathrm{~Hz}$, equivalent to 2500 boundary-layer turnover times at the lowest operating velocity. Between them, a readjustment period of $3 \mathrm{~min}$ is given for the response of the system to reach a nearly constant value. The SNR of these measurements is found within the range $1<\sigma / \mu<5$, where $\sigma$ and $\mu$ are the standard deviation and the mean value of the signal, respectively. Once the run is over, the wind tunnel is turned off and the balance is set to idle for another period of half an hour between runs. The aforementioned procedure is repeated five times for the results to achieve statistical significance.

Alternatively to the linear regression method described above, it is possible to implement a PID controller to drive the blower as a function of the pitching moment. The acquisition process is, therefore, expedited since only one data point is taken at each $R e$, as opposed to 5. The accuracy of the controller is dependent on the sensitivity of the blower and resolution of the duty cycle.

\subsection{Uncertainty estimates}

This analysis follows the rules established by the International Bureau of Weights and Measures for evaluating and expressing uncertainty in measurement (BIPM et al. 2008). The complete uncertainty budget for the friction velocity $U_{\tau}$ is given in Fig. 8 as a function of the Reynolds number. It factors the

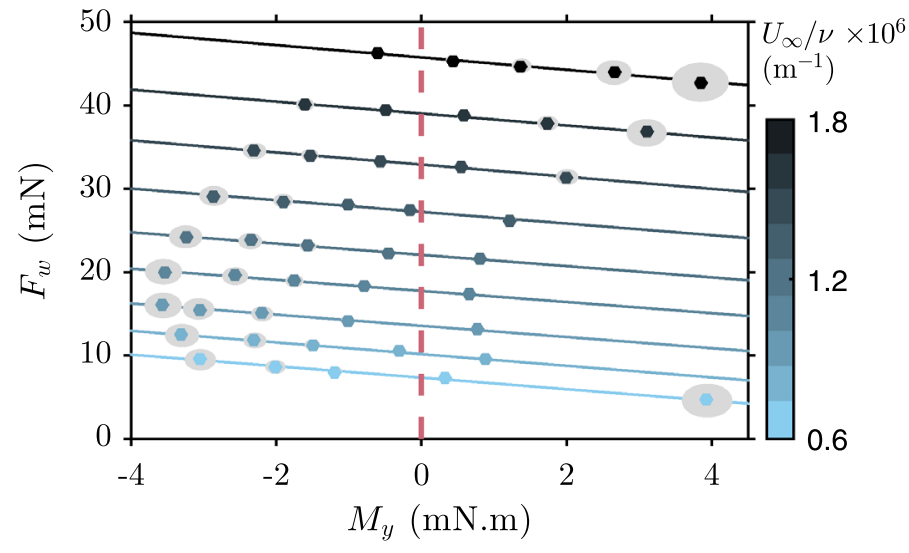

Fig. 7 Method to evaluate the WSS. On the left: at each Re number, indicated by the colormap, five data points were acquired across a range of pressures $p_{\mathrm{b}}$. Linear curves were then fit to each individual set infer the drag value for which the pitching moment is null, indi-

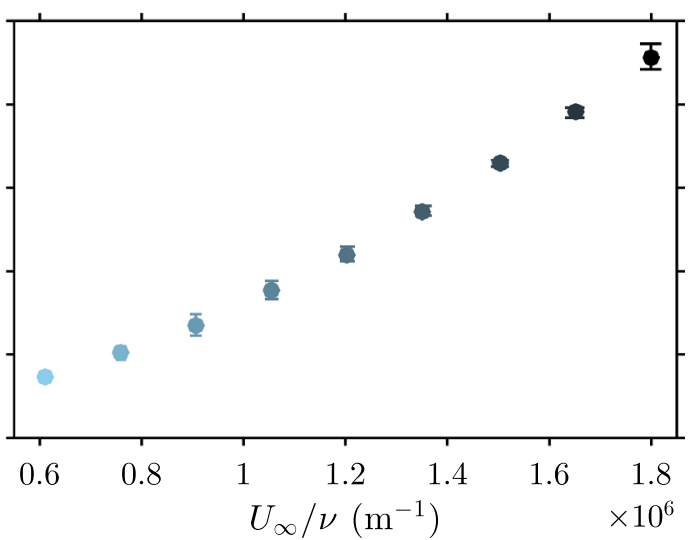

cating the absence of a vertical net force. The grey-shaded regions show the uncertainty associated with the measurements, addressed in Sect. 3.4. On the right: intercept of the linear regressions and corresponding uncertainty bar as a function of unit $R e$ 


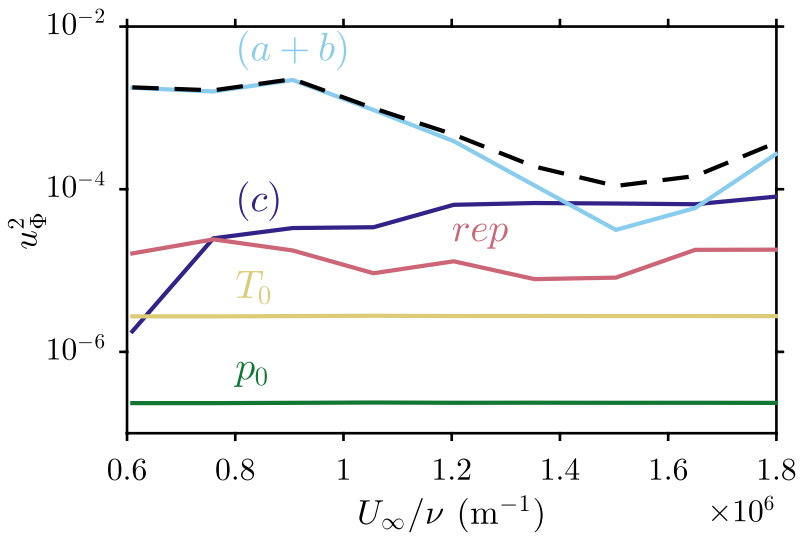

Fig. 8 Uncertainty budget. Breakdown of the total variance of $U_{\tau}$, black dashed-line, as a function of Reynolds number. The light-blue line shows the contribution both due to calibration errors and the wall-drag evaluation method, $u_{(\mathrm{a}+\mathrm{b})}$; dark-blue line refers to the error induced by the inclination of the FE, $u_{(\mathrm{c})}$; the green and yellow curves show, respectively, the uncertainties associated with pressure and temperature, $u_{\text {(d) }}$; the red line reflects the repeatability of the measurements, $u_{(\mathrm{e})}$

most important contributions, including (a) calibration errors, (b) the inference method of WSS, (c) the inclination of the FE, (d) determination of air properties, temperature and pressure, and (e) precision of the measurements.

By definition $U_{\tau}=\sqrt{\tau_{\mathrm{w}} / \rho_{0}}$; the WSS is expressed as $\tau_{\mathrm{w}}=F_{\mathrm{w}} / S$ and the air density is obtained via the ideal gas law $\rho_{0}=p_{0} /\left(R T_{0}\right)$, where $R=287.05 \mathrm{~J} \mathrm{~kg}^{-1} \mathrm{~K}^{-1}$ is the specific gas constant, $p_{0}$ is the atmospheric pressure and $T_{0}$ is the room temperature. Hence, $U_{\tau}\left(F_{\mathrm{w}}, T_{0}, p_{0}\right)$ is a function of three independent variables. Assuming all input quantities are uncorrelated, the combined uncertainty of $U_{\tau}$ is the positive square root of the variance $u_{U_{\tau}}^{2}$, written as

$$
\begin{aligned}
& u_{U_{\tau}}^{2}=\underbrace{\left(\frac{\partial U_{\tau}}{\partial F_{\mathrm{w}}}\right)^{2} u_{F_{\mathrm{w}}}^{2}}_{(\mathrm{a})+(\mathrm{b})+(\mathrm{c})}+ \\
& \underbrace{\left(\frac{\partial U_{\tau}}{\partial T_{0}}\right)^{2} u_{T_{0}}^{2}+\left(\frac{\partial U_{\tau}}{\partial p_{0}}\right)^{2} u_{p_{0}}^{2}}_{(\mathrm{d})}+\underbrace{u_{\mathrm{rep}}^{2}}_{(\mathrm{e})},
\end{aligned}
$$

where $u_{\Phi}$ is the standard uncertainty of the arbitrary variable $\Phi$.

Contributions ( $a$ ) and (b) The first term on the RHS of Eq. 8 is the combination of sources (a), (b) and (c), which are inherent to the working principle of the balance. Particularly, (a) and (b) cannot be decoupled; the calibration error $u_{(\text {a) }}$ propagates through the linear regression and is reflected in the uncertainty estimate of the Y-intercept, from here on denoted $u_{(\mathrm{a}+\mathrm{b})} . u_{(\mathrm{a})}$ is determined by multiplying the readings of the force transducers by the covariance matrix of the calibration coefficients $\left(\bar{X}^{T} W^{j} \bar{X}\right)_{5 \times 5}^{-1}$, defined in Sect. 3.2. Accordingly,

$\left.u_{(\mathrm{a}) j}=X\left(\bar{X}^{T} W^{j} \bar{X}\right)^{-1}\right) X^{T}$,

where $u_{(\mathrm{a})}$ is the uncertainty associated with the load component $j$ (drag or pitch), $\bar{X}$ is the averaged model matrix of the calibration, $W^{j}$ is the weighting matrix for $j$ and $X$ is the vector containing the readings of the force transducers, written according to the model form expressed in Eq. 3. Potential errors induced by wire misalignments, friction of the pulley and weight application are all factored into the covariance matrix $W^{j}$. Further details can be found in Reis et al. (2013). $u_{(\mathrm{a}+\mathrm{b})}$ amounts to approximately $97 \%$ of the total variance at low Re but only $30 \%$ at higher values. In absolute terms, its magnitude is fairly independent of the loading conditions, so its relative significance diminishes with $R e$ as opposed to that of $u_{(\mathrm{c})}$, which increases monotonically to become the primary source of uncertainty.

Contribution (c) Surface inclination causes the pressure-based wall-normal load $F_{\mathrm{n}}$ to be projected in the streamwise direction. Similar to WSS, this error contribution is a flow-driven phenomenon which manifests as an offset in skin-friction coefficient (Baars et al. 2016). Provided a constant tilt angle, it can be corrected for, but the additional DOF of the present device rules out this possibility. It thus stands as a source of bias error that must be included in the uncertainty budget, $u_{(\mathrm{c})}$. The streamwise projection $F_{\mathrm{w}}^{\prime}=F_{\mathrm{n}} \sin (\alpha)$, where $\alpha$ is the tilt angle of the FE. Then it follows that

$u_{(c)}^{2}=\underbrace{\left(\frac{\partial F_{\mathrm{w}}^{\prime}}{\partial F_{\mathrm{n}}}\right)^{2} u_{F_{\mathrm{n}}}^{2}}_{=0}+\left(\frac{\partial F_{\mathrm{w}}^{\prime}}{\partial \alpha}\right)^{2} u_{\alpha}^{2}$.

The flow-exposed surface is mounted parallel to the principal flow direction, thus $\sin (\alpha)=0$ and $u_{\mathrm{F}_{\mathrm{n}}}$ can readily be ignored. In turn, the accuracy of the digital spirit level $\left(0.1^{\circ}\right)$ in addition to the maximum predicted rotation of the $\mathrm{FE}$ $\left(0.075^{\circ}\right)$ yields $u_{\alpha}=\sqrt{0.1^{2}+0.075^{2}} \simeq 0.125^{\circ}$. The second term of Eq. 10 can be rewritten as $\left.\left[\Delta p_{\mathrm{d}} S \cos (\alpha)\right)\right]^{2} u_{\alpha}^{2}$, where $\Delta p_{\mathrm{d}}$ is the pressure difference at which wall drag is evaluated. Considering that buoyancy forces, no matter how small, entail the existence of a net vertical force when the pitching moment is cancelled out, $\Delta p_{\mathrm{d}}$ will depend on the flow conditions. Specifically, the latter increases with $R e$, hence the trend $u_{(\mathrm{c})}$ is exhibits in Fig. 8.

Contribution (d) Air density is function of the atmospheric pressure and room temperature, $\rho_{0}\left(p_{0}, T_{0}\right)$. The first was acquired at the start and finish of the experiment, 
using a weather station accurate to within $100 \mathrm{~Pa}$. The mean value was then assigned to $p_{0}$, so it follows that its uncertainty includes not only the accuracy of the measurement system but also the range of the fluctuations; $u_{p_{0}}=\sqrt{\left(P_{0_{\text {acc }}}\right)^{2}+\left(P_{0_{\max }}-P_{0_{\min }}\right)^{2}}$. The freestream temperature, on the other hand, was continuously monitored using a T-type Omega thermocouple with an accuracy of $0.5^{\circ}$. A similar system was dedicated to measure temperature inside the balance.

Differential pressures were monitored via three Furness FCO510 micromanometers (model 2) with full-scale (FS) ranges of 200 and $2000 \mathrm{~Pa}$. These have a reported accuracy of \pm 1 digit below $20 \mathrm{~Pa}$ and $0.25 \%$ of the reading above this threshold. The freestream dynamic pressure $q_{0}$ is acquired via a Pitot-static tube positioned above the FE, aligned with its leading edge. Following the assumption of a thin boundary layer, potential wall-normal pressure gradients are neglected and the pressure over the flowexposed surface of the FE is taken to be equal to the local static pressure. The static port is then shared with a second manometer, by means of a T-junction, to monitor the pressure difference between the top and underside of the FE $\Delta p$. Additionally, the streamwise pressure gradient $\partial p / \partial x$ is estimated by measuring the pressure drop between the aforementioned Pitot-static tube and another Pitot probe mounted far upstream, assuming a linear growth of the boundary layer. Note that from these quantities solely $q_{0}$ represents a source error in $C_{\mathrm{f}}\left(F_{\mathrm{w}}, q_{0}\right)$. Despite playing a role in the uncertainty estimation of $F_{\mathrm{w}}$, that of $\Delta p$ is not factored in this analysis (refer to Eq. 10).

\section{Results}

Measurements of skin friction for both the smooth and the rough walls were acquired following the procedure described in Sect. 3.3. A total of 5 runs were performed in subsequent days for freestream velocities ranging between $U_{0}=10$ and $26 \mathrm{~ms}^{-1}$ in increments of $2 \mathrm{~ms}^{-1}$. Pre- and post-calibrations were performed for each configuration without notable discrepancies, owing to the long-term stability of this device.

\subsection{Smooth wall}

Values of WSS inferred from hot-wire anemometry of the boundary-layer profile were used as the standard of comparison for the FE measurements. The results, given in Fig. 9, show an excellent agreement between these techniques, especially for $R e_{\theta}$ over $5 \times 10^{3}\left(U_{0}=14 \mathrm{~ms}^{-1}\right)$ where the relative difference between them falls below $2 \%$. For the most part, the disparity of the data at low freestream velocities may be explained by the increased uncertainty

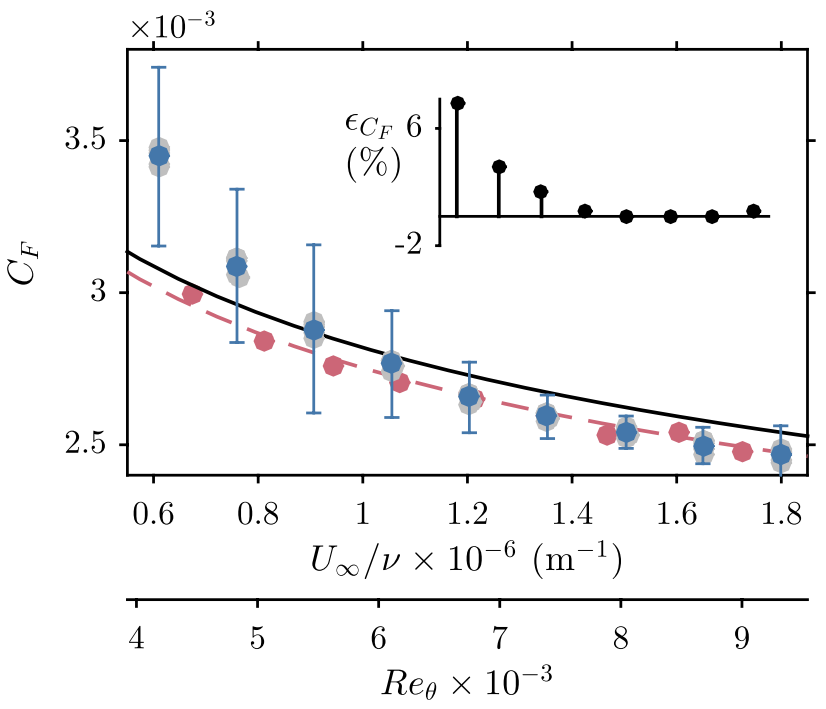

Fig. 9 Smooth-wall friction coefficient. $C_{\mathrm{F}}$ as a function of unit $R e$ and $R e_{\theta}$. Red circles show the values inferred from hot-wire anemometry; the dash line is a power curve fit though those points. Blue circles are the mean FE measurements; the error bars indicate the magnitude of the uncertainty. The black solid line is the smoothwall skin-friction correlation for $R e_{\theta}$ from Österlund et al. (2000), $C_{\mathrm{F}}=2\left((1 / \kappa) \ln \left(R e_{\theta}\right)+4.08\right)^{-2}$. The inset shows the relative discrepancy of the FE measurements from the values of hot-wire anemometry

in the measurement; with the exception of the first data point, whose error bar does not encompass the power curve fit through the hot-wire values. In absolute terms, the magnitude of the force discrepancy is approximately $0.92 \mathrm{mN}$, which makes it remarkably difficult to identify its cause. This could either be an indication of additional error sources which were not accounted for, or of poorly estimated uncertainty contributions. A similar behaviour is observed on the skin-friction measurements of Baars et al. (2016), who suggested that it could be related to secondary loads acting on non-parallel surfaces to the flow and to the projection of the pressure-based force in the streamwise direction that could not be fully eliminated. From these potential contributors, the first was not considered in the uncertainty analysis presented in Sect. 3.4. Additionally, it is plausible to assume some residual coupling of the vertical net force, despite the measures to work around this limitation.

The smooth-wall skin-friction relationship of Österlund et al. (2000) is also included in Fig. 9 for comparison. While the values of $C_{\mathrm{F}}$ obtained from hot wire share the same trend, they are generally lower by $2 \%$. This is also true for the FE measurements at higher Reynolds numbers, plausibly for the reasons discussed in Sect. 3: the streamwise pressure gradient in the contraction and tripping conditions. 


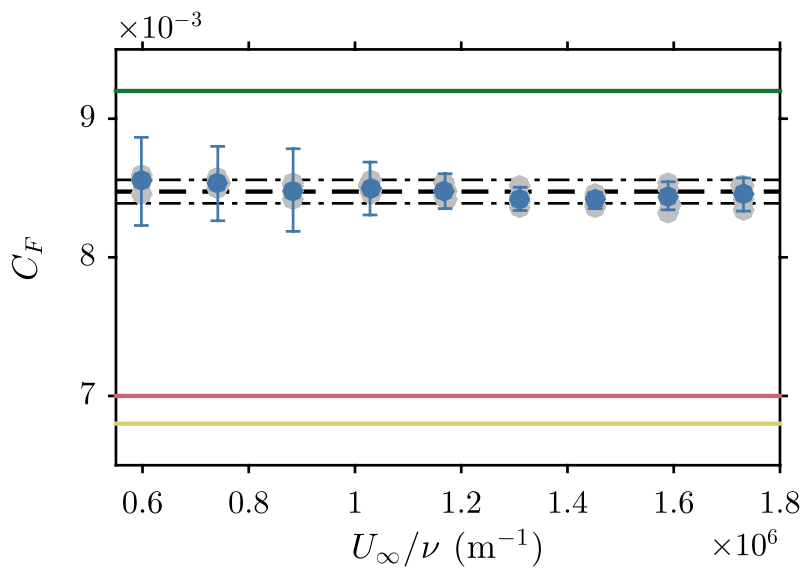

Fig. 10 Rough-wall friction coefficient. Blue circles are the mean FE measurements of $C_{\mathrm{F}}$ for each unit $R e$; the error bars indicate the magnitude of the uncertainty. The average value across $R e$ is indicated by the black dash line which is bounded by black dash-dotted lines corresponding to $\pm 1 \%$ of the mean. The yellow line shows the value inferred from the Reynolds shear stress by Cheng and Castro (2002); and the red and green indicate the values obtained by Claus et al. (2012) using a pivot-type FE and pressure-tapped roughness obstacles, respectively

\subsection{Rough wall}

$C_{\mathrm{F}}$ measurements of the rough-wall boundary layer are shown in Fig. 10. Values obtained using the present FE take a nearly constant value (to within $1 \%$ ) across the entire range of $R e$, which indicates the flow reaches the fully rough regime farther upstream of its location. Here, we determine the equivalent sand-grain roughness $k_{\mathrm{s}}$, normalised to viscous units $k_{\mathrm{s}}^{+}=k_{\mathrm{s}} U_{\tau} / \nu$, based on the boundary-layer thickness reported by Cheng and Castro (2002) for the same flow conditions, $\delta=0.12 \mathrm{~m}$ at $U_{0}=10 \mathrm{~m} / \mathrm{s}$. Assuming the functional form for the viscous-scaled mean velocity profile $U^{+}$of Coles (1956), the downward shift relative to the smooth-wall case $\Delta U^{+}=13.8$. This yields $k_{\mathrm{s}}^{+}=1070$ that is well above the empirical threshold $k_{\mathrm{s}}^{+} \geq 80$ from which the flow/surface becomes fully rough (Jiménez 2004). The test-retest variability of the measurements appears to have worsened compared to the smooth wall, revealed by the spread of individual (grey) data points around the mean. At high $R e$, it becomes in fact the largest uncertainty contribution. Additional estimates of $C_{\mathrm{F}}$ are included in the same figure, specifically that inferred from cross-wire anemometry of the boundary-layer profile by Cheng and Castro (2002), and those obtained by Claus et al. (2012) using a pivot-type FE and pressure-tapped roughness obstacles. Overall, the wall drag over the staggered array of cubes is substantially higher compared to the smooth wall and the disparity between the estimates is in the order of $25 \%$.

Claus et al. (2012) highlighted the marked discrepancy between their measurements and advanced the hypothesis that, while trends with $R e$ are correct, the uncertainty in the pressure data could undermine the validity of the form-drag estimate. They further argue, by comparing the results from both techniques, that a lower $C_{\mathrm{F}}$ of the FE would imply a negative friction drag contribution. On these grounds, and given the quoted uncertainty of their FE (Krogstad and Efros 2010), they concluded the absolute pressure-drag value is likely overestimated. The present measurements support this observation, as the mean skin-friction coefficient $\overline{C_{\mathrm{F}}}=8.45 \times 10^{-3}$ is still $8 \%$ lower than that of the pressure data. However, they do not agree with the FE estimate of Claus et al. (2012) which is $17 \%$ lower in comparison. To explain this discrepancy, it would be required to carry out a comprehensive uncertainty analysis of their measurements, otherwise we can only speculate. Two plausible sources of error are contemplated here. The first is related to the streamwise pressure gradient, which could be neglected in terms of flow development (based on the acceleration parameter), but still stands as a source of buoyancy effects. Naturally, the suction wind tunnel where these experiments were conducted imposes a favourable pressure gradient in the freestream and over the flow-exposed surfaces. Since the distribution on the underside of the FE is likely to be uniform, the pressure difference across $\Delta p$ becomes larger downstream. As small as it may be, this effect induces a moment opposite to the action of the WSS that could result in an apparent drag reduction considering the setup of Claus et al. (2012). Another potential source of uncertainty has to do with roughness-induced pressure inhomogeneities within the canopy. When the roughness length scale is comparable to the size of the FE, the integrated vertical pressure force is likely to lie off-centre, thus creating a spurious moment around the pivot point with similar consequences to that mentioned above depending on the obstacle distribution.

Cheng and Castro (2002) reported wind tunnel measurements over geometrically identical staggered arrays of cubes, 10 and $20 \mathrm{~mm}$ tall. They evaluate $C_{\mathrm{F}}$ for both surfaces from spatially averaged shear stress profiles, using cross-wire anemometry. For the largest case, they further provide an estimate based on the pressure distribution over a unit area, and verified that the shear stress in the inertial subrange is roughly $21 \%$ lower in comparison-it possibly does not reflect the spatial anisotropy within the canopy. In view of this observation, Reynolds and Castro (2008) defined a correction factor for the WSS of staggered arrays of cubes based on the shear stress profile $\left(U_{\tau}=1.12 \sqrt{-\overline{u v}}\right)$, which Placidi and Ganapathisubramani (2015) extended to arbitrary cubical arrays with varying frontal and plan solidities. The underlying assumption to this approximation is that the ratio between the shear stress above the canopy and the total surface stress remains constant, or it changes marginally with fetch. Accordingly, we can use the value 
of $C_{\mathrm{F}}$ obtained by Cheng and Castro (2002) taken at the same fetch as the present experiment, shown in Fig. 10, to obtain a corrected estimate of $C_{\mathrm{F}}$ to compare against the FE measurement. This yields a skin-friction coefficient of $8.8 \times 10^{-3}$ that is only $4 \%$ higher. A slight overestimation could either be explained by the uncertainty associated with the form-drag data, which was not provided, or by limitations of the aforementioned assumption. Nevertheless, the agreement is remarkable.

\section{Conclusion}

In this paper, we proposed a self-contained FE design based on the parallel-shift linkage. Different from traditional arrangements, one set of flexures was replaced by single bending-beam transducers to monitor the streamwise load. The position of the FE is then fixed and it does not require preloading. Additionally, pitching moment transducers in liaison with a centrifugal blower successfully mitigate the potential coupling of the vertical load due to residual transverse sensitivity of the force transducers. A second-order polynomial in two variables was used to calibrate the balance. The typical standard error of the fitting is $\hat{\sigma}_{1}=0.057 \mathrm{mN}$ and $\hat{\sigma}_{2}=0.006 \mathrm{~m} \mathrm{Nm}$ for drag and pitch, respectively. The FE showed a notable long-term stability, as discrepancies between the results obtained with calibrations carried out 1 month apart are generally smaller than $0.5 \%$.

Measurements of skin-friction coefficient $C_{\mathrm{F}}$ for a smooth-wall boundary layer agree with values inferred from hot-wire anemometry to within $2 \%$ for $R e_{\theta}>4 \times 10^{3}$. A detailed uncertainty analysis revealed the most important error contributions are related to the calibration and drag evaluation method, as well as the tilt angle of the FE. In contrast, the test-retest variability amounted to a small fraction of the total uncertainty budget. This experiment further considered the flow over a rough surface of staggered distributed cubes with large relative height $(\delta / h \simeq 10)$ and 0.25 solidity fraction. Results showed the boundary layer is fully rough at the measurement location, since $C_{\mathrm{F}}$ takes a nearly constant value across the range of Reynolds number and $k_{\mathrm{s}}^{+}=1070$ at the lowest operating velocity $\left(U_{0}=10 \mathrm{~ms}^{-1}\right)$. The mean skin-friction coefficient $\overline{C_{\mathrm{F}}}=8.45 \times 10^{-3}$ was compared against estimates from previous studies. Of particular interest is the FE measurement of Claus et al. (2012), which underestimates the present value by $17 \%$, highlighting the importance of buoyancy effects on pivot-type balances. Although less straight forward, it was possible to establish a comparison with estimates obtained from cross-wire anemometry of the boundary layer, provided by Cheng and Castro (2002), assuming $U_{\tau}=1.12 \sqrt{-\overline{u v}}$ (Reynolds and Castro 2008). In this case, the relative difference in $C_{\mathrm{F}}$ is approximately $4 \%$.

Acknowledgements This work was financially supported by the Engineering and Physical Sciences Research Council (EPSRC) through Grants EP/P009638/1 and EP/P021476/1. We would also like to acknowledge the contribution to the design of Dr. Roeland De Kat from the faculty of Engineering and the Environment of the University of Southampton. Any relevant data produced in this study will be made available upon publication of this manuscript.

Open Access This article is distributed under the terms of the Creative Commons Attribution 4.0 International License (http://creativeco mmons.org/licenses/by/4.0/), which permits unrestricted use, distribution, and reproduction in any medium, provided you give appropriate credit to the original author(s) and the source, provide a link to the Creative Commons license, and indicate if changes were made.

\section{References}

Acharya M, Bornstein J, Escudier MP, Vokurka V (1985) Development of a floating element for the measurement of surface shear stress. AIAA J 23:410-415

Allen JMH (1977) Experimental study of error sources in skin-friction balance measurements. J Fluids Eng 99:197-204

Allen JMH (1980) Improved sensing element for skin-friction balance measurements. AIAA J 18:1342-1345

Antonia RA, Krogstad PÅ (2001) Turbulence structure in boundary layers over different types of surface roughness. Fluid Dyn Res 28:139-157

Antonia RA, Luxton RE (1971) The response of a turbulent boundary layer to a step change in surface roughness part 1 . Smooth to rough. J Fluid Mech 48:721-761

Baars WJ, Squire DT, Talluru KM, Abbassi MR, Hutchins N, Marusic I (2016) Wall-drag measurements of smooth- and rough-wall turbulent boundary layers using a floating element. Exp Fluids 57(5):90

BIPM, IEC, IFCC, ILAC, ISO, IUPAC, IUPAP, OIML (2008) Guide to the expression of uncertainty in measurement. Joint Committee for Guides in Metrology 10:2008, GUM 1995 with minor corrections

Brown KC, Joubert PN (1969) The measurement of skin friction in turbulent boundary layers with adverse pressure gradients. J Fluid Mech 35(4):737-757

Chauhan KA, Monkewitz PA, Nagib HM (2009) Criteria for assessing experiments in zero pressure gradient boundary layers. Fluid Dyn Res 41(021):404

Cheng H, Castro IP (2002) Near wall flow over urban-like roughness. Bound Layer Meteorol 104:229-259

Claus J, Krogstad PÅ, Castro IP (2012) Some measurements of surface drag in urban-type boundary layers at various wind angles. Bound Layer Meteorol 145:407-422

Clauser FH (1956) Advances in applied mechanics, vol 4. The turbulent boundary layer. Elsevier, Amsterdam, pp 1-51

Coles D (1953) Measurements in the boundary layer on a smooth flat plate in supersonic flow. $\mathrm{PhD}$ thesis, California Institute of Technology, Pasadena

Coles D (1956) The law of the wake in the turbulent boundary layer. J Fluid Mech 1:191-226

Dhawan S (1951) Direct measurements of skin friction. PhD thesis, California Institute of Technology, Pasadena 
Dogan E, Hanson RE, Ganapathisubramani B (2016) Interactions of large-scale free-stream turbulence with turbulent boundary layers. J Fluid Mech 802:79-107

Esteban LB, Dogan E, Rodríguez-López E, Ganapathisubramani B (2017) Skin-friction measurements in a turbulent boundary layer under the influence of free-stream turbulence. Exp Fluids $58: 115$

Frei D, Thomann H (1980) Direct measurements of skin friction in a turbulent boundary layer with a strong adverse pressure gradient. J Fluid Mech 101:79-95

Hirt F, Zurfluh U, Thomann H (1986) Skin friction balances for large pressure gradients. Exp Fluids 4:296-300

Jiménez J (2004) Turbulent flows over rough walls. Annu Rev Fluid Mech 36:173-196

Johnson H, Parker PA, Landman D (2010) Calibration designs for nonmonolithic wind tunnel force balances. In: Proceedings of the 7th international symposium on strain-gauge balances, 10-13 May, Williamsburg

Kempf G (1929) Neue ergebnisse der widerstandsforschung. Werft, Reederei, Hafen, pp 234-239

Krogstad PÅ, Efros V (2010) Rough wall skin friction measurements using a high resolution surface balance. Int J Heat Fluid Flow 31:429-433 (6th international symposium on turbulence and shear flow phenomena)

Krogstad PÅ, Antonia RA, Browne LWB (1992) Comparison between rough- and smooth-wall turbulent boundary layers. J Fluid Mech 245:599-617

Medjnoun T, Vanderwel C, Ganapathisubramani B (2018) Characteristics of turbulent boundary layers over smooth surfaces with spanwise heterogeneities. J Fluid Mech 838:516-543

Musker AJ (1979) Explicit expression for the smooth wall velocity distribution in a turbulent boundary layer. AIAA J 17:655-657

O'Donnel FB Jr (1964) A study of the effect of floating-element misalignment on skin-friction-balance accuracy. Defense research laboratory report, University of Texas

Ozarapoglu V (1973) Measurements in incompressible turbulent flows. $\mathrm{PhD}$ thesis, Université Laval, Québec

Pailhas G, Barricau P, Touvet Y, Perret L (2009) Friction measurement in zero and adverse pressure gradient boundary layer using oil droplet interferometric method. Exp Fluids 47:195-207
Placidi M, Ganapathisubramani B (2015) Effects of frontal and plan solidities on aerodynamic parameters and the roughness sublayer in turbulent boundary layers. J Fluid Mech 782:541-566

Placidi M, Ganapathisubramani B (2018) Turbulent flow over large roughness elements: effect of frontal and plan solidity on turbulence statistics and structure. Bound Layer Meteorol 167:99-121

Reis MLCC, Castro RM, Mello OAF (2013) Calibration uncertainty estimation of a strain-gage external balance. Measurement 46:24-33

Reynolds RT, Castro IP (2008) Measurements in an urban-type boundary layer. Exp Fluids 45:141-156

Rodríguez-López E, Bruce PJK, Buxton ORH (2015) A robust postprocessing method to determine skin friction in turbulent boundary layers from the velocity profile. Exp Fluids 56:68

Rodríguez-López E, Bruce PJK, Buxton ORH (2016) On the formation mechanisms of artificially generated high reynolds number turbulent boundary layers. Bound Layer Meteorol 160:201-224

Rodríguez-López E, Bruce PJK, Buxton ORH (2017) Experimental measurement of wall shear stress in strongly disrupted flows. J Turbul 18:271-290

Schmidt MA, Howe RT, Senturia SD, Haritonidis JH (1988) Design and calibration of a microfabricated floating-element shear-stress sensor. IEEE Trans Electron Devices 35:750-757

Sheplak M, Cattafesta LN, Nishida T, McGinley CB (2004) Mems shear stress sensors: promise and progress. In: Proceedings of the 24th AIAA aerodynamic measurement technology and ground testing conference, ARC

Österlund JM, Johansson AV, Nagib HM, Hites MH (2000) A note on the overlap region in turbulent boundary layers. Phys Fluids 12(1): $1-4$

Strutz T (2016) Data fitting and uncertainty, 2nd edn. Springer Vieweg, Berlin (chap 3)

Townsend AA (1976) Structure of turbulent shear flow, 2nd edn. Cambridge University Press, Cambridge

Winter KG (1979) An outline of the techniques available for the measurement of skin friction in turbulent boundary layers. Prog Aerosp Sci 18:1-57

Publisher's Note Springer Nature remains neutral with regard to jurisdictional claims in published maps and institutional affiliations. 